\title{
Do IgA, IgE, and IgG avidity tests have any value in the diagnosis of toxoplasma infection in pregnancy?
}

\author{
D Ashburn, A W L Joss, T H Pennington, D O Ho-Yen
}

\begin{abstract}
Aim-To determine the value of tests for specific IgA, IgE, and IgG avidity in diagnosing Toxoplasma gondii infection during pregnancy.

Methods-In a retrospective study, current serological tests (dye test and three IgM assays with different sensitivities) were compared with immunosorbent agglutination assays (ISAGA) for specific IgA and IgE and an IgG avidity enzyme linked immunosorbent assay (ELISA). Patient group 1 comprised six women with definite or probable infection during pregnancy determined by congenital toxoplasmosis or laboratory results. Group 2 comprised seven women infected during or before 11 pregnancies (two consecutive pregnancies in two patients and three in a third).

Results-One patient in group 1 seroconverted during pregnancy. IgA ISAGA and avidity confirmed acute infection when confirmatory IgM ELISA remained negative. In five of six patients from group 1, IgA and IgE ISAGA and avidity confirmed acute infection. In group 2, the dye test titre was raised in seven of 11 pregnancies (six of seven patients). Specific IgM and IgA were positive during all 11 pregnancies. IgE ISAGA was positive in only four of 11 pregnancies (three of seven patients), but negative results in the remainder may exclude acute infection. High avidity antibodies indicative of past infection were found in four of 11 pregnancies (two of seven patients).

Conclusions-Each test improved diagnosis or timing of infection but no single test was ideal. The IgA ISAGA was sensitive and detected seroconversion. Positive IgE ISAGA and low avidity both confirmed infection, whereas negative IgE may exclude acute infection. High avidity diagnosed past infection but persistence of low avidity reduced its value to differentiate acute and past infection. Further studies with larger patient groups are needed to determine the optimum diagnostic strategy. These techniques are valuable in complementing existing tests.

(f Clin Pathol 1998;51:312-315)
\end{abstract}

Keywords: immunoglobulin avidity; toxoplasmosis; pregnancy

When a mother acquires primary Toxoplasma gondii infection during pregnancy, congenital infection may result, with severe consequences for the fetus. ${ }^{1}$ However, as the risk of fetal infection is very small when maternal infection occurs before conception, it is important to make a precise estimation of the timing of maternal infection. ${ }^{2}$ Serological diagnosis of toxoplasma infection is usually based on fourfold increases in IgG titre and demonstration of specific $\operatorname{IgM} .{ }^{3}$ As rising titres are seldom demonstrated outside an antenatal screening programme, ${ }^{4}$ detection of specific $\operatorname{IgM}$ is often used to indicate acute infection. However, detection of $\operatorname{IgM}$ may be protracted, ${ }^{3}$ and therefore interpretation of results is dependent on the sensitivity of the $\operatorname{IgM}$ technique, ${ }^{56}$ and with the use of current tests it may not be possible to determine the onset of maternal infection accurately.

In order to overcome these difficulties several new techniques have been developed. Immunoglobulin A $(\operatorname{IgA})$ is produced as part of the acute phase response, and has been found to be of value in the investigation of cytomegalovirus infection. ${ }^{7}$ Toxoplasma specific IgA has been measured both by enzyme linked immunosorbent assay (ELISA) ${ }^{48}$ and immunosorbent agglutination assay (ISAGA), ${ }^{49}$ in the sera of patients with acquired primary infection. Following infection it is claimed that specific $\operatorname{IgA}$ production parallels that of specific $\operatorname{IgM},{ }^{10}$ or lags slightly behind IgM. ${ }^{9}$ Toxoplasma specific $\mathrm{IgE}$ is also detected early after infection. ${ }^{11-13}$ Results suggest that the duration of detectable specific IgE may be shorter than IgM or IgA. ${ }^{11-13}$ While measurement of specific IgA and IgE may prove useful in improving the assessment of timing of infection, there is wide patient to patient variation in their detection. ${ }^{814}$ Measurement of IgG avidity represents a different approach to antibody studies, assessing the interaction between antibody and antigen. ${ }^{15}$ In early infection low avidity antibodies predominate, whereas later high avidity antibodies are more common. ${ }^{16}$ Measurement of IgG avidity has been applied to several viral infections, ${ }^{15}$ and to toxoplasma infection, ${ }^{15}{ }^{17}$ in which it has been used to time maternal infection during pregnancy. ${ }^{19}$

In this study, to determine whether newer techniques have any value in the diagnosis of toxoplasma infection during pregnancy, IgA and IgE ISAGAs and an IgG avidity assay based on an in-house IgG ELISA were used. The results were compared with those obtained using current tests (dye test, two different IgM ELISAs with different sensitivity, and an IgM ISAGA).

\section{Methods}

In a retrospective study, toxoplasma antibodies were measured in 43 sera from 17 pregnancies in 13 pregnant women. Sera had been tested using
Accepted for publication 5 February 1998 
current tests: in-house $\operatorname{IgG}^{20}$ and $\operatorname{IgM}^{21}$ ELISAs, and a micromodification of the dye test. ${ }^{22}$ Toxoplasma specific IgM positive results were confirmed using a commercial assay, Toxonostika ELISA-IgM (Organon Technika, Cambridge, UK). Those that were negative in the screening test or not confirmed were also tested using the more sensitive Toxo-ISAGA IgM (BioMerieux, Marcy l'Etoile, France). Patients were divided into two groups, determined by the presence of congenital toxoplasmosis and the results of current serological tests.

Group 1 consisted of six patients with definite or probable infection during pregnancy. The outcome of pregnancy was congenital toxoplasmosis in three cases. In two of the three cases there were rising dye test titres, raised titres ( $\geqslant 250 \mathrm{IU} / \mathrm{ml}$ ) in the third, and IgM was confirmed positive in all. Two other cases were included on the basis of raised dye test titres and confirmed IgM. One pregnancy had a normal outcome, but was included because the mother had cervical lymphadenopathy, a raised dye test titre, and specific IgM detectable in the latter stages of pregnancy. This combination of clinical and laboratory data indicates infection during pregnancy. The second pregnancy ended in fetal death which may have been caused by toxoplasma, but no tissues were available for confirmation. The remaining patient seroconverted during pregnancy, but again the outcome was normal. In this patient IgM was not confirmed by Toxonostika ELISA IgM, but was positive by the more sensitive Toxo-ISAGA.

Group 2 comprised seven women with past or possible acute infection during 11 pregnancies (two consecutive pregnancies in two women, and three in a third). These were further subdivided into groups $2 \mathrm{a}$ and $2 \mathrm{~b}$.

Group 2a comprised women with only a single pregnancy or the first of two or three consecutive pregnancies. Raised dye test titres were measured in all four single pregnancies and during the first pregnancy in two other women. Toxonostika ELISA IgM was also positive in two of four single pregnancies and in all three first pregnancies, but toxoplasma specific IgM was detected by one or more of the methods used in all group 2a pregnancies. Specific IgM was detected early in all four single pregnancies and-in the absence of symptoms or rising dye test titres-it was therefore not possible to determine whether infection occurred before or after conception.

Group $2 \mathrm{~b}$ included the second pregnancy in three women and the third in one of these. Although the dye test titre was normal in the first specimens taken during each of the consecutive pregnancies, the Toxonostika ELISA-M was positive. Thus group 2 shows that similar laboratory results may be obtained in both past infection and possible acute infection.

All sera were tested using in-house immunosorbent agglutination assays for specific $\operatorname{Ig} \mathrm{A}^{23}$ and IgE. ${ }^{12}$ Briefly, round bottomed microtitre plates (Immulon 1, Dynex Technologies, Billingshurst, UK) were coated with capture antibody (Dako, Copenhagen, Denmark), antihuman IgA ( $\alpha$ chain specific; $2 \mu \mathrm{g} / \mathrm{ml}$ ) or anti- human $\operatorname{IgE}$ ( $\varepsilon$ chain specific; $2.4 \mu \mathrm{g} / \mathrm{ml}$ ) for the IgA and IgE ISAGA, respectively. Patients' sera $(100 \mu \mathrm{l})$ diluted $1 / 100$ in phosphate buffered saline (PBS), $\mathrm{pH} 7.2$, were added to triplicate wells. After incubation for two hours at $37^{\circ} \mathrm{C}$ wells were washed with PBS plus $0.05 \%$ Tween 20 (pH 7.2) (PBST) and then with PBS alone. Formaldehyde fixed tachyzoites $\left(1.25 \times 10^{7} /\right.$ $\mathrm{ml})$ were added to each of the triplicate $(100$, 150 , and $200 \mu \mathrm{l}$ ) wells and incubated overnight at $37^{\circ} \mathrm{C}$. Results were scored $0-4$ in each well relative to negative (no agglutination) and positive (agglutination) controls. The scores of triplicate wells were accumulated to produce the ISAGA matrix (maximum 12). A matrix of $\leqslant 2$ was negative, 3-4 equivocal, 5-9 positive, and $\geqslant 10$ strong positive.

IgG avidity was measured using the in-house IgG ELISA under conditions similar to a published method. ${ }^{19}$ Flat bottomed microtitre plates (Virion B, Shield Diagnostics, Dundee, UK) were coated overnight with toxoplasma antigen optimally diluted in a $10 \%$ solution of ELISA coating buffer (Don Whitley Scientific, Shipley, W Yorkshire, UK). After aspirating the plates, they were dried at $37^{\circ} \mathrm{C}$ for four hours and stored at $-20^{\circ} \mathrm{C}$ until use. Patients' sera were diluted with PBST in fourfold steps to produce five dilutions: $1 / 50,1 / 200,1 / 800$, $1 / 3200,1 / 12800$. The four highest dilutions $(1 / 200-1 / 12800)$ were added $(100 \mu$ l per well $)$ to rows $\mathrm{A}-\mathrm{D}$ and the four lowest to rows $\mathrm{E}-\mathrm{H}$. After incubation at room temperature for one hour, the plates were washed as follows: rows A-D, $3 \times 5$ minutes with PBST; rows $\mathrm{E}-\mathrm{H} 3 \times$ 5 minutes with urea wash solution (6 M urea in PBST). All wells were then washed a further three times with PBST. Conjugateantihuman IgG-alkaline phosphatase (Sigma, Poole, Dorset, UK) — was added (100 $\mu$ l per well) at optimal dilution. After incubation for one hour at room temperature, plates were washed with PBST and substrate added (Sigma phosphatase substrate) diluted in ELISA substrate buffer (Don Whitley Scientific). After 30 minutes, the optical density was measured at $405 \mathrm{~nm}$ using a Dynatech MR-5000 plate reader (Dynex Technologies). Dilution curves were plotted for the urea washed and PBST washed wells and avidity calculated as the ratio of titres measured (urea:PBST) at the threshold of 0.2 absorbance units. Low, borderline, and high avidity was $<15 \%, 15-30 \%$, and $>30 \%$ respectively.

\section{Results}

Group 1 comprised six women with definite or probable evidence of $T$ gondii infection during pregnancy. In one patient, who seroconverted during pregnancy, toxoplasma specific IgM was detected only by the BAM-ELISA and Toxo-ISAGA. Positive IgA ISAGA results and low avidity IgG were also measured, but IgE ISAGA failed to diagnose acute infection in this patient. In the other five patients from group 1, positive results in the IgA ISAGA, IgE ISAGA, and low avidity IgG (table 1) confirmed a diagnosis of acute infection.

In group $2 \mathrm{a}$ and $2 \mathrm{~b}$, the IgA ISAGA was positive during all pregnancies (table 1 ). The 
Table 1 Serological results obtained during 17 pregnancies

\begin{tabular}{|c|c|c|c|c|c|c|c|c|c|}
\hline \multirow[b]{2}{*}{ Grou } & & \multirow[b]{2}{*}{ Patient No } & \multirow[b]{2}{*}{$\begin{array}{l}\text { Raised dye test titre } \\
(\geqslant 250 \mathrm{IU} / \mathrm{ml})\end{array}$} & \multirow[b]{2}{*}{$B A M-E L I S A$} & \multicolumn{4}{|c|}{ Positive results in: } & \multirow[b]{2}{*}{$\operatorname{Ig} G$ avidity ${ }^{*}$} \\
\hline & & & & & $\begin{array}{l}\text { Toxonostika } \\
\text { ELISA IgM }\end{array}$ & Toxo-ISAGA $M$ & $\operatorname{Ig} A$ & $\operatorname{Ig} E$ & \\
\hline \multirow{6}{*}{\multicolumn{2}{|c|}{1}} & 1 & + & + & + & ND & + & + & $\mathrm{L}$ \\
\hline & & 2 & + & + & + & & + & + & \\
\hline & & 3 & + & + & + & ND & + & + & $\mathrm{L}$ \\
\hline & & 4 & + & + & + & ND & + & + & $\mathrm{L}$ \\
\hline & & 5 & + & + & + & ND & + & + & $\mathrm{L}$ \\
\hline & & 6 & + & + & - & + & + & - & $\mathrm{L}$ \\
\hline \multirow[t]{11}{*}{2} & (a) Single/first & 7 & + & + & + & ND & + & + & B \\
\hline & pregnancy & 8 & + & + & + & ND & + & - & $\mathrm{H}$ \\
\hline & & 9 & + & + & - & + & + & - & $\mathrm{L}$ \\
\hline & & 10 & + & - & - & + & + & - & $\mathrm{H}$ \\
\hline & & 11 & - & + & + & ND & + & - & B \\
\hline & & 12 & + & - & + & ND & + & + & $\mathrm{L}$ \\
\hline & & 13 & + & + & + & ND & + & + & $\mathrm{H}$ \\
\hline & (b) Consecutive & 11 & - & + & + & ND & + & - & $\mathrm{L}$ \\
\hline & pregnancies & 12 & - & - & + & + & + & - & $\mathrm{L}$ \\
\hline & & & - & - & - & + & + & - & $\mathrm{L}$ \\
\hline & & 13 & - & + & + & ND & + & + & $\mathrm{H}$ \\
\hline
\end{tabular}

Results shown are those in the first sample or post-seroconversion sample tested.

+ , positive; -, negative; ND, not done; ${ }^{\star} \mathrm{L}$, low (<15\%); B, borderline $(15-30 \%)$; H, high $(>30 \%)$.

IgE ISAGA was positive in only four of 11 pregnancies from three of seven patients; three of these were during the first or a single pregnancy (group 2a) and the fourth from a consecutive pregnancy (group 2b). Negative results could possibly have been used to exclude acute infection in four of seven and three of four group $2 \mathrm{a}$ and $2 \mathrm{~b}$ pregnancies, respectively. Avidity measurement was less useful, as high avidity antibodies were measured in only three of seven group 2 a patients and in one of four group $2 b$ pregnancies. Furthermore IgG avidity did not mature from low to high avidity in two patients between their first pregnancy in group $2 \mathrm{a}$ and their second or third in group $2 \mathrm{~b}$. In contrast to group 1 patients, simultaneous positive results for IgM, IgA, IgE, and IgG antibodies of low avidity were measured in only one group $2 \mathrm{a}$ patient during her first pregnancy. These results suggest that infection occurred during this pregnancy. Therefore if all of the results from all tests were used, a correct timing of the infection could be achieved.

\section{Discussion}

Protracted detection of raised levels of specific IgM makes timing infection in pregnancy difficult. ${ }^{1}$ Diagnosis of infection during pregnancy is therefore often based on comparison of results from two or three IgM tests. ${ }^{3}$ In this laboratory, the less sensitive Toxonostika ELISA IgM assay is most useful in the confirmation of onset of infection within a period $\leqslant 1-2$ trimesters. ${ }^{6}$ Patients were divided into two groups according to the likelihood of infection. Thus it was possible to evaluate the three relatively new tests on their ability to improve on the existing IgM based strategy.

It has been claimed that specific IgA is detected early after infection but also disappears rapidly. ${ }^{24}{ }^{25}$ In our study specific IgA was detected in the first post-seroconversion sample in one patient, confirming its rapid appearance after infection. However, the IgA ISAGA was also positive in all other pregnancies from patients in both groups. Persistence of raised levels of specific IgA has been reported previously. ${ }^{425}$ Our results of patients followed through consecutive pregnancies (group 2) confirm that, like specific IgM, specific IgA positive results may persist for prolonged periods. Therefore the IgA ISAGA was not able to differentiate between acute and past infection. This may reflect the enhanced sensitivity of ISAGA over ELISA methods ${ }^{4}$; use of a less sensitive IgA ELISA may be more appropriate to improve timing of infection and should be investigated.

In other studies, ${ }^{11}{ }^{14}$ detection of specific $\operatorname{IgE}$ was associated with toxoplasma infection during pregnancy in 33 of 37 women. In our study, toxoplasma specific IgE was detected in five of six patients from group 1, but not in the patient who seroconverted, confirming the results of the Toxonostika ELISA IgM. In our study, there was no evidence of congenital toxoplasmosis in this patient and therefore negative $\operatorname{IgE}$ ISAGA results may indicate a favourable outcome of infection during pregnancy. Further testing of more patients is necessary to confirm the prognostic value of a negative IgE ISAGA result. Specific IgE was not detected in eight of 11 pregnancies from five of seven patients from group 2. It has been suggested that specific IgE remains detectable for less than four months after infection. ${ }^{11}$ Therefore, negative results may be useful to exclude acute infection. However, because IgE assays may lack sensitivity, ${ }^{11}{ }^{12}$ results of the IgE ISAGA should be interpreted in conjunction with results of other tests.

Measurement of IgG avidity has previously been applied to determination of duration of infection, ${ }^{18} 1926$ and to identifying pregnancies at risk of congenital toxoplasmosis. ${ }^{19}$ One study ${ }^{19}$ included 11 women who seroconverted during pregnancy. All had low avidity antibodies, which in 10 of 11 women matured to high avidity within six months. The remaining patient still produced low avidity antibodies after 11 months. This is different from the second study in which the mean avidity result was low both in sera collected less than 20 weeks and between 20 to 52 weeks after infection. ${ }^{26}$ The discrepancy between these studies is 
probably because in the first, ${ }^{19}$ results were correlated with the time elapsed since the last seronegative sample compared with the estimated time since infection. ${ }^{26}$ Nevertheless, in both studies high avidity was measured in past infection. In our study, all patients from group 1 , including one who seroconverted, had low avidity antibodies. Low avidity antibodies were also measured in five of 11 pregnancies from three of seven patients in group 2. As three of these were from group $2 b$, and infection was therefore before pregnancy (second pregnancies of two patients and the third pregnancy of another), the value of low avidity antibody measurement to confirm acute infection may be less than previously suggested. ${ }^{19}{ }^{26}$ Furthermore persistence of low avidity antibody, like persistent IgM, may complicate diagnosis.

These findings may indicate variation in immune response. ${ }^{17}$ Persistent borderline avidity antibody has also been reported in nonpregnant patients with lymphadenopathy present for over six months. ${ }^{18}$ High avidity antibodies in four pregnancies, three of which were confirmed as IgM positive, were used to diagnose past infection. Thus the IgG avidity ELISA has proved less useful in our studies than previously described. ${ }^{1926}$ This may be attributed to the study size or to the patients tested. Our inclusion of sera collected from three women with persistent $\operatorname{IgM}$ during consecutive pregnancies may have introduced a sampling bias absent from other studies. ${ }^{1926}$ Alternatively, the discrepancies may have been a result of difference between assay procedures, although this was not indicated by our results in non-pregnant patients or comparison with a commercial kit. ${ }^{27}$

The three women with persistent IgM during consecutive pregnancies may represent a group not previously studied. ${ }^{19}$ How frequently they occur is not known. However, they are an important group in which to assess the ability of relatively new tests to differentiate between acute and past infection. All the consecutive pregnancies in group $2 b$ were known to have had past infection, yet neither specific IgA, IgE, nor IgG avidity results suggested past infection in every case. Therefore, like specific IgM tests, these have to be interpreted with caution during pregnancy.

In conclusion, the IgA ISAGA is sensitive and can be used to detect seroconversion. Positive IgE ISAGA results and low avidity antibodies were both able to confirm acute infection. The absence of specific $\operatorname{IgE}$ in patients from group 2 may have been useful to exclude acute infection. Measurement of high avidity antibodies was also used to diagnose past infection. However, persistence of low avidity antibodies meant that the IgG avidity ELISA was a less useful discriminator of current from past infection than previously suggested. More conclusive interpretation of the data may be limited by the small number of patients tested, and further studies with a larger population are necessary to determine the optimum diagnostic strategy. However, each of these techniques provided additional information to diagnose and time infection during pregnancy, although no single test was ideal. Therefore the value of specific IgA, IgE, or IgG avidity tests is to augment rather than replace the current tests.

We are grateful to the Scottish Home and Health Department (grant No K/MRS/50/C1828) and the European Network on Congenital Toxoplasmosis, working group on serological diagnosis (Biomed 1 programme PL921572 of the commission of the European Union), for funding. We would also like to thank Ms Debbie Gilham for secretarial assistance, and the users of the Scottish Toxoplasma Reference Laboratory for their support.

1 Remington JS, Desmonts G. Toxoplasmosis. In: Remington $\mathrm{S}$, Klein JO, eds. Infectious diseases of the fetus and newborn infant. Philadelphia: WB Saunders, 1990:89-195.

2 Desmonts G, Couvreur J. Toxoplasmosis in pregnancy and its transmission to the fetus. Bull N Y Acad Med 1974;50:146-59.

3 Joss AWL. Diagnosis. In: Ho-Yen DO, Joss AWL, eds. Human toxoplasmosis. Oxford: Oxford University Press, 1992:79-118.

4 Patel B, Young Y, Duffy K, et al. Immunoglobulin-A detection and the investigation of clinical toxoplasmosis. $7 \mathrm{Med}$ Microbiol 1993;38:286-92.

5 Skinner LJ, Chatterton JMW, Joss AWL, et al. The use of an IgM immunosorbent agglutination assay to diagnose

6 Ashburn D, Evans R, Skinner LJ, et al. Comparison of relative uses of commercial assays for Toxoplasma gondii IgM antibodies. F Clin Pathol 1992;45:483-6.

7 Crange MP, Kennedy D, Venters JL, et al. IgA responses during human cytomegalovirus infection in cardiac transplant recipients: concurrent detection of $\operatorname{IgA}$ and $\operatorname{IgM}$ antiglobulins. Serodiagn Immunother Infect Dis 1988;2:301-9.

8 Francis JM, Joynson DH. Duration of specific immunoglobulin A antibody following acute toxoplasmosis as determined by enzyme immunoassay and immunosorbent agglutination assay. Eur f Clin Microbiol Infect Dis 1993;12: $556-9$.

9 Bessières $\mathrm{MH}$, Roques $\mathrm{C}$, Berrebi $\mathrm{A}$, et al. IgA antibody response during acquired and congenital toxoplasmosis. $\mathcal{F}$ Clin Pathol 1992;45:605-8.

10 Wong SY, Remington JS. Toxoplasmosis in pregnancy. Clin Infect Dis 1994;18:853-61.

11 Pinon JM, Toubas D, Marx C, et al. Detection of specific immunoglobulin $\mathrm{E}$ in patients with toxoplasmosis. F Clin Microbiol 1990;28:1739-43.

12 Ashburn D, Joss AW, Pennington TH, et al. Specificity and usefulness of an IgE immunosorbent agglutination assay usefulness of an IgE immunosorbent agglutinat

13 Montoya JG, Remington JS. Studies on the serodiagnosis of toxoplasmic lymphadenitis. Clin Infect Dis 1995;20:781-9.

14 Wong SY, Hajdu MP, Ramirez R, et al. Role of specific immunoglobulin $\mathrm{E}$ in diagnosis of acute toxoplasma infection and toxoplasmosis. F Clin Microbiol 1993;31:2952-9.

15 Hedman K, Lappalainen M, Söderlund M, et al. Avidity of IgG in serodiagnosis of infectious diseases. Rev Med Micro 1993;4:123-9.

16 Holliman RE. Recent developments in the diagnosis of toxoplasmosis. Serodiagn Immunother Infect Dis 1994;6:5-16.

17 Holliman RE, Raymond R, Renton N, et al. The diagnosis of toxoplasmosis using IgG avidity. Epidemiol Infect 1994;112:399-408.

18 Joynson DHM, Payne RA, Rawal BK. Potential role of IgG avidity for diagnosing toxoplasmosis. F Clin Pathol 1990;43: 1032-3.

19 Lappalainen M, Koskela P, Koskiniemi M, et al. Toxoplasmosis acquired during pregnancy: improved serodiagnosis based on avidity of IgG. F Infect Dis 1993;167:691-7.

20 Joss AWL, Skinner LJ, Chatterton JMW, et al. Toxoplasmosis: effectiveness of enzyme immunoassay Toxoplasmosis: effectiveness of enzy

21 Joss AWL, Skinner LJ, Moir IL, et al. Biotin-labelled antigen screening test for toxoplasma IgM antibody. 7 Clin Pathol 1989;42:206-9.

22 Williams KAB, Scott JM, Macfarlane DE, et al. Congenital toxoplasmosis: a prospective survey in the west of Scotland. F Infect 1981;3:219-29.

23 Ashburn D, Joss AW, Ho-Yen D, et al. The value of toxoplasma specific IgA in diagnosis. F Clin Pathol 1995;48: 689.

24 Decoster A, Darcy F, Caron A, et al. Anti-P30 IgA antibodies as prenatal markers of congenital toxoplasma infection. Clin Exp Immunol 1992;87:310-15.

25 Foudrinier F, Marx-Chemla C, Aubert D, et al. Value of specific immunoglobulin A detection by two immunocapture assays in the diagnosis of toxoplasmosis. Eur $\mathcal{F}$ Clin Microbiol Infect Dis 1995;14:585-90.

26 Jenum PA, Stray-Pedersen B, Gundersen AG. Improved diagnosis of primary Toxoplasma gondii infection in early pregnancy by determination of antitoxoplasma immunoglobulin G avidity. F Clin Microbiol 1997;35:1972-7.

27 Ashburn D. The relevance of IgA and IgE assays, IgG avidity and western blotting in the diagnosis of toxoplasma infection. Aberdeen: University of Aberdeen, 1996. (PhD thesis.) 